\title{
Association of Knee Extensor Muscle Strength and Cardiorespiratory Fitness With Bone Stiffness in Japanese Adults: A Cross-sectional Study
}

\author{
Takahisa Ohta ${ }^{1,2,3}$, Junzo Nagashima ${ }^{2,4}$, Wataru Fukuda ${ }^{1,2,3}$, Hiroyuki Sasai ${ }^{3}$, and Naokata Ishii ${ }^{5}$ \\ ${ }^{1}$ Graduate School of Health and Sport Science, Nippon Sport Science University, Tokyo, Japan \\ ${ }^{2}$ Yokohama Sports Medical Center, Nissan Stadium, Yokohama, Japan \\ ${ }^{3}$ Research Team for Promoting Independence and Mental Health, Tokyo Metropolitan Institute of Gerontology, Tokyo, Japan \\ ${ }^{4}$ Division of Cardiology, Department of Internal Medicine, St. Marianna University School of Medicine, Kanagawa, Japan \\ ${ }^{5}$ Department of Life Sciences, Graduate School of Arts and Sciences, The University of Tokyo, Japan
}

Received December 2, 2020; accepted March 30, 2021; released online April 10, 2021

\begin{abstract}
Background: Knee extensor muscle strength and cardiorespiratory fitness (CRF) are major components of physical fitness. Because the interactive association of knee extensor muscle strength and CRF with bone health remains unclear, we aimed to investigate such association in Japanese adults.

Methods: Altogether, 8,829 Japanese adults (3,731 men and 5,098 women) aged $\geq 45$ years completed the maximum voluntary knee extension test, submaximal exercise test, medical examination, and a questionnaire on lifestyle habits. Using an osteo-sono assessment index, low bone stiffness tendency was defined as $80 \%$ under the young-adults mean. Multivariable odds ratios (ORs) and 95\% confidence intervals (CIs) were calculated after confounder adjustment.

Results: Overall, 542 men (14.5\%) and 978 women (19.2\%) had low bone stiffness tendency. We observed an inverse association between muscle strength and low bone stiffness tendency after adjustment for CRF in both sexes $(P$ for linear trend $<0.001)$. Compared with the lowest CRF, the multivariable ORs for low bone stiffness tendency in the highest CRF were 0.47 (95\% CI, 0.36-0.62) for men and 1.05 (95\% CI, 0.82-1.35) for post-menopausal women $(P<0.001$ and $P=0.704$, respectively). No interactive association between muscle strength and CRF for low bone stiffness tendency existed in both sexes and irrespective of menopausal status.

Conclusion: Knee extensor muscle strength and CRF were associated additively, not synergistically, with bone health. Maintaining high levels of both physical fitness components may improve musculoskeletal health in the cohort. The relationship between physical fitness and bone status should be longitudinally investigated in the future.
\end{abstract}

Key words: knee extensor muscle strength; cardiorespiratory fitness; bone stiffness; quantitative ultrasound

Copyright $\odot 2021$ Takahisa Ohta et al. This is an open access article distributed under the terms of Creative Commons Attribution License, which permits unrestricted use, distribution, and reproduction in any medium, provided the original author and source are credited.

\section{INTRODUCTION}

Osteoporosis is characterized by low bone strength and an increased risk of bone fractures, ${ }^{1}$ which is highly associated with mortality., ${ }^{2,3}$ Moreover, as osteoporosis imposes a heavy economic burden on the gross domestic product, ${ }^{4}$ it is widely recognized as a serious public health concern not only in Japan but also in other aged societies. Currently, Japan experiences one of the most serious situations in $\mathrm{Asia}^{5}$ because the estimated number of patients with osteoporosis exceeds 12.8 million (men: 3 million; women: 9.8 million). ${ }^{6}$ To minimize the detrimental effects of osteoporotic fractures on a patient's quality of life, early detection of low bone strength and preventive interventions preferably based on risk stratification are strongly needed.

The established risk factors for low bone strength are advanced age, female sex, genetic factors, low body weight, and physical inactivity, including low physical fitness represented as cardiorespiratory fitness (CRF) and/or grip strength. ${ }^{7,8}$ Compared to low CRF, high CRF represents an odds ratio of 0.29 (95\% confidence interval $[\mathrm{CI}], 0.12-0.71$ ) for the femoral neck Tscore $\leq 2.5$. $^{7}$ The hazard ratio for osteoporotic fracture per $5 \mathrm{~kg}$ reduction in hand grip strength was 1.49 (95\% CI, 1.18-1.95) in a 10-year prospective cohort study. ${ }^{8}$ Maintaining high levels of physical fitness, assessed via CRF and/or hand grip strength, is a key factor for preventing low bone strength, which consequently lowers the risk of osteoporosis development.

Grip strength is often used as an indicator of muscle strength because it is valid and reliable for whole-body muscle strength. ${ }^{9}$ However, its use is controversial since it cannot be measured for antigravity muscles, such as the knee extensor muscle. ${ }^{10}$ As knee extensor muscle strength predicts the risk of falls and mobility, it can be used as a better indicator of bone strength. ${ }^{11,12}$ To our 
knowledge, it remains unclear whether knee extensor muscle strength is beneficially associated with bone strength. Moreover, CRF is also one of the predictors for low bone strength. ${ }^{7}$ However, information on the association between knee extensor muscle strength and bone strength according to varying levels of CRF is limited.

This study aimed to investigate the interactive associations of knee extensor muscle strength and CRF with bone stiffness as a marker of bone strength in Japanese adults. The findings from this cross-sectional study will allow us to design future longitudinal and clinical studies to investigate the preventive effect of various strategies for osteoporosis and possibly result in a decrease in osteoporotic fractures.

\section{METHODS}

\section{Design, setting, and participants}

This study was a cross-sectional analysis on the association of knee extensor muscle strength and CRF with bone stiffness. The eligibility criteria were: i) Sport Program Service (SPS, explained below) participants between April 1998 to July 2019, ii) aged $\geq 45$ years, and iii) participants who had undergone bone stiffness measurements. SPS is a comprehensive medical checkup program primarily examining various domains of physical fitness held at the Yokohama Sports Medical Center. The SPS was initiated in April 1998 to improve the health status of people living or working in Yokohama City. Participants voluntarily apply to the service through the center's website, and public information is published by the local government. This service receives an average of 10 people daily, totaling to 1,500 people annually. Participants eligible to use the service are those aged between 18 and 65 years, living or working in Yokohama City, and paid 15,000 JPY (approximately $\$ 142$ in 2020); aged $>65$ years and paid 7,500 JPY (\$71); and not living and working in Yokohama City, aged $<65$ years old, and paid 17,000 JPY (\$161) and 8,500 JPY (\$80). All data were selected at a single timepoint when participants first joined the service.

Prior to joining the SPS, all participants provided their informed consent for data use. This study was conducted according to the Declaration of Helsinki, and the protocol was approved by the Ethics Committee of the Yokohama Sports Medical Center (K-2019-07).

\section{Measurements}

\section{Medical checkup}

Height and body weight of barefoot participants were measured using a calibrated height-weight scale (WB-510; Tanita Co., Tokyo, Japan). Body mass index (BMI) was calculated as body weight/height ${ }^{2}\left(\mathrm{~kg} / \mathrm{m}^{2}\right)$. After a 5-minute sitting position on a chair and medical examination by a physician, resting blood pressure was measured through the Riva-Rocci Korotkov method using a mercury sphygmomanometer. Blood glucose, total cholesterol, high-density lipoprotein (HDL) cholesterol, lowdensity lipoprotein (LDL) cholesterol, triglycerides (TG), alkaline phosphatase (ALP), and uric acid (UA) were sampled after 12hour fasting and analyzed with Roche INTEGRA 400 plus (Roche International Ltd., Basle, Switzerland). Data on habitual alcohol drinking (yes or no), habitual smoking status (yes or no), and menopausal status (yes or no) were obtained through a selfreported questionnaire.

\section{Bone stiffness and definition of low bone stiffness tendency}

The generally known method for assessing bone strength is dual energy X-ray absorptiometry (DXA), which is commonly used to diagnose osteoporosis. As DXA emits radiation to assess the bone, there are problems, such as radiation exposure, costliness, being time-consuming, access to the place where DXA is set up, and the need for radiologists. In contrast, quantitative ultrasound (QUS), which is used to assess bone stiffness as an objective marker of bone strength, is well established in Japan. ${ }^{6}$ Compared to DXA, QUS results in better outcomes for the patients as it avoids exposure to radiation and is inexpensive, highly portable, and efficient. Therefore, in this study, bone stiffness was assessed using a QUS device (AOS-100NW; Hitachi Aloka Medical, Ltd., Mitaka, Tokyo, Japan) that has a high correlation with other QUS devices or DXA $(r=0.804, P<0.001) .{ }^{13,14}$ Maintenance and calibration were performed to preserve the quality of the results once per month. The method of measurement was as follows: the participants sat on a chair barefoot with their knees bent at $90^{\circ}$. A device was placed on their right calcaneal, and gel was applied on the membranes. The measurement was performed once for approximately 30 seconds. Bone stiffness was expressed using the osteo-sono assessment index, which was calculated using the speed of sound and transmission index ${ }^{15}$ and was highly reproducible. ${ }^{13}$ Young adult mean (YAM) was calculated based on average age of 20 to 44 years as $100 \% .^{16}$ Low bone stiffness tendency was defined as $80 \%$ under the YAM (YAM80\%), according to the 2011 Japanese guideline for prevention and treatment of osteoporosis-executive summary. ${ }^{6}$ Similarly, a $70 \%$ under the YAM (YAM70\%) was also calculated to analyze sensitivity.

\section{Physical fitness test}

Knee extensor muscle strength, which assessed maximum voluntary knee extension torque, was measured using an isokinetic dynamometer (Cybex Humac Norm 770; Computer Sports Medicine Inc., Stoughton, MA, USA). The measurement process was as follows: the participants sat so that their knee and hip joint formed a right angle and performed warm-ups. Thereafter, the participants exerted isokinetic maximum voluntary knee extension at 60 degrees/s at least twice. Overall, the participants performed three repetitions with a 30 -second break. The maximum value was considered as knee extensor strength (Nm) and adjusted by the participant's own body weight $(\mathrm{Nm} / \mathrm{kg})$.

CRF was assessed by applying physical working capacity at $75 \%$ of the maximum heart rate $\left(\mathrm{PWC} 75 \% \mathrm{HR}_{\max }\right){ }^{17}$ which was highly correlated with maximal oxygen uptake $(r=0.942),{ }^{18}$ using the submaximal graded exercise test method on an electronic bicycle ergometer (The Multi Exercise Test System, ML-1800, Fukuda-Denshi, Tokyo, Japan). On the graded exercise test, the rate of loading $(10-60 \mathrm{~W} / \mathrm{min})$, which was an individualized ramp protocol, was decided by experts based on the participants' age and habitual aerobic exercise. The target heart rate was set at $75 \%$ of the estimated maximum heart rate (220 minus age), and the test was ended upon reaching target value. Additionally, the participants could end the test when an abnormal electrocardiogram result (ST depression or frequent occurrence of the extrasystole) was confirmed by the cardiologists or when they could not pedal with the designated rhythm (50 rpm) while showing poor physical condition. Most participants ended the test at approximately $10 \mathrm{~min}$. 
Ohta $\mathrm{T}$, et al.

Table 1. Participant characteristics according to knee extensor muscle strength

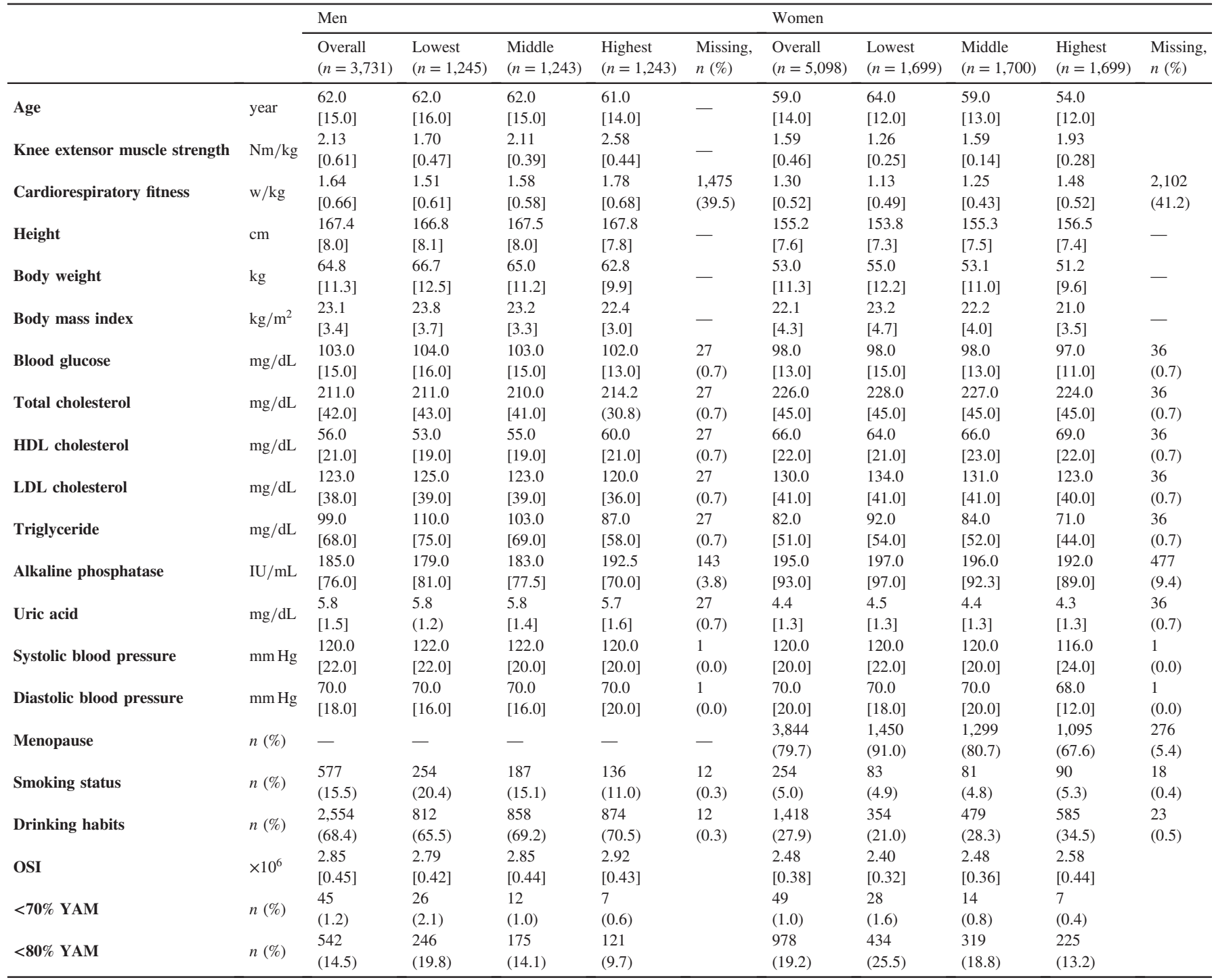

HDL, high-density lipoprotein; LDL, low-density lipoprotein cholesterol; OSI, osteo-sono assessment index; and YAM, young-adult mean.

Data are presented as median [interquartile ranges], mean (standard deviation), and number (percentage) unless specified.

\section{Statistical analysis}

Due to clear sex and age differences in the prevalence of osteoporosis, ${ }^{19}$ the participants were segregated by sex and age group (aged 45-54, 55-64, and $\geq 65$ years), and categorized into tertiles based on knee extensor muscle strength. Thereafter, each age category was combined based on muscle strength, and new groups were created with age-adjusted tertiles. Continuous and categorical variables were expressed as median (interquartile rages) or mean (standard deviation), and percentage, respectively.

To reduce potential biases due to incomplete data, ${ }^{20}$ missing data were treated with multiple imputation methods using SPSS (IBM, Inc., Chicago, IL, USA) by creating a random number through the Markov chain Monte Carlo algorithm, wherein 20 para-complete datasets were produced. ${ }^{21}$ Blood glucose, total cholesterol, HDL cholesterol, LDL cholesterol, TG, ALP, and UA were used as auxiliary variables to account for the missing data. The auxiliary variables were not used in the main analysis. The 20 datasets were integrated with the standard Rubin's technique. Every missing value is presented in Table 1.
To assess the association of each covariate, knee extensor muscle strength, and CRF with bone stiffness, univariable odds ratios and 95\% CIs were calculated using a logistic regression model. The covariates included age, ${ }^{22}$ smoking status, ${ }^{23}$ drinking habits, ${ }^{24} \mathrm{BMI},{ }^{25}$ systolic blood pressure, ${ }^{26}$ and menopause. ${ }^{23}$

To evaluate the association between knee extensor muscle strength and low bone stiffness tendency, multivariable odds ratios and 95\% CIs were calculated using the lowest knee extensor muscle strength group as reference after adjusting for smoking status, drinking habits, BMI, systolic blood pressure, and menopause (for women only). In the mutually adjusted final model, CRF was entered into the final model. Moreover, a continuous variable of knee extensor muscle strength was used in another model to test linearity. The same analyses were repeated with CRF as the primary exposure and knee extensor muscle strength as the final covariate. To consider the moderating effect of menopausal status, a menopause-stratified multivariable analysis was also performed. 
To evaluate the interactive association of knee extensor muscle strength and CRF with low bone stiffness tendency, a CRFstratified (below or above the median) multivariable analysis was performed. Odds ratios and 95\% CIs were calculated similarly as mentioned above, and the interaction term (knee extensor muscle strength $* \mathrm{CRF}$ ) was entered into the models. A menopausestratified multivariable analysis was also conducted.

To verify the validity of the applicable standards for low bone stiffness tendency, the following two sensitivity analyses were performed. First, we repeated the primary analysis by changing the definition of low bone stiffness tendency from $80 \%$ under the YAM to $70 \%$ under the YAM. Second, a complete-case analysis was conducted to distinguish findings with and without considering missing values. All sensitivity analysis data are shown in the eTable 1 , eTable 2, eTable 3, eTable 4, eTable 5, eTable 6, and eTable 7.

All statistical analyses were performed using the SPSS version 25. A $P$-value $<0.05$ was considered significant.

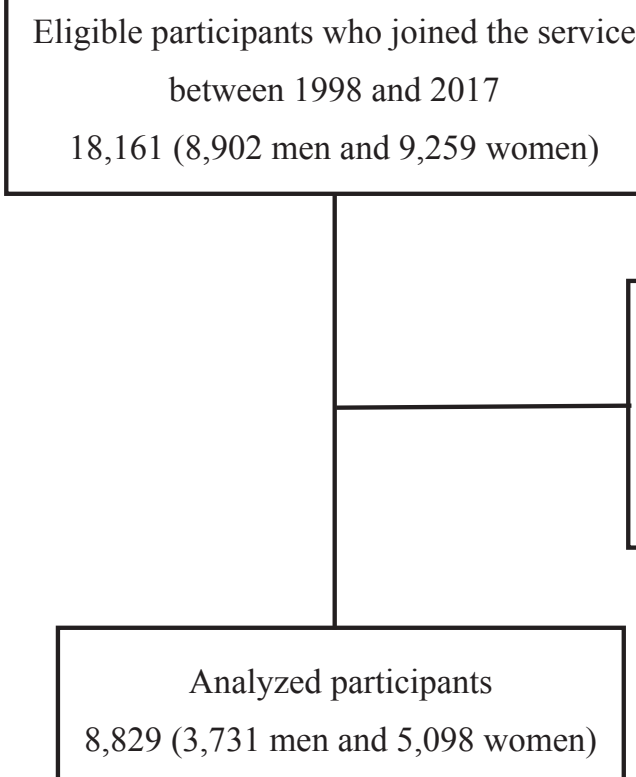

Figure 1. Flowchart of participant selection

Table 2. Univariate associations of each covariate and two main exposure variables with low bone stiffness tendency

\begin{tabular}{|c|c|c|c|c|c|c|c|c|c|c|c|}
\hline \multirow{2}{*}{ Variables } & \multirow{2}{*}{ Units } & \multicolumn{5}{|l|}{ Men } & \multicolumn{5}{|l|}{ Women } \\
\hline & & Participant & Frequency & Prevalence $^{\mathrm{a}}$ & Odds ratio $(95 \% \mathrm{CI})$ & $P$ value & Participant & Frequency & Prevalence $^{\mathrm{a}}$ & Odds ratio $(95 \% \mathrm{CI})$ & $P$ value \\
\hline Age & Years & 3,731 & 542 & 145.3 & $1.02(1.01-1.03)$ & $<0.001$ & 5,098 & 978 & 191.8 & $1.10(1.09-1.11)$ & $<0.001$ \\
\hline \multirow{2}{*}{ Smoking status } & No & 3,153 & 422 & 133.8 & 1.00 (reference) & \multirow{2}{*}{$<0.001$} & 4,844 & 930 & 192.0 & 1.00 (reference) & \multirow{2}{*}{0.907} \\
\hline & Yes & 578 & 120 & 207.6 & $1.70(1.35-2.13)$ & & 254 & 48 & 189.0 & $0.98(0.71-1.35)$ & \\
\hline \multirow{3}{*}{ Drinking habits } & No & 1,180 & 202 & 171.2 & 1.00 (reference) & \multirow{3}{*}{0.002} & 3,674 & 779 & 212.0 & 1.00 (reference) & \multirow{3}{*}{$<0.001$} \\
\hline & Yes & 2,551 & 340 & 133.3 & $0.74(0.61-0.90)$ & & 1,424 & 199 & 139.7 & $0.61(0.51-0.72)$ & \\
\hline & $<18.5$ & 109 & 34 & 311.9 & $2.58(2.08-3.20)$ & & 448 & 141 & 318.3 & $1.89(1.53-2.35)$ & \\
\hline \multirow[t]{2}{*}{ Body mass index } & $18.5-25.0$ & 2,704 & 405 & 149.8 & 1.00 (reference) & \multirow[t]{2}{*}{0.029} & 3,602 & 712 & 197.9 & 1.00 (reference) & \multirow[t]{2}{*}{$<0.001$} \\
\hline & $\geq 25.0$ & 908 & 103 & 113.4 & $0.73(0.65-0.82)$ & & 1,053 & 122 & 116.1 & $0.53(0.43-0.65)$ & \\
\hline \multirow{2}{*}{ Menopause } & No & - & - & & - & - & 1,039 & 19 & 18.3 & 1.00 (reference) & \multirow{2}{*}{$<0.001$} \\
\hline & Yes & - & - & & - & - & 4,060 & 959 & 236.2 & $16.33(10.31-25.88)$ & \\
\hline \multirow{4}{*}{$\begin{array}{l}\text { Knee extensor } \\
\text { muscle strength }\end{array}$} & Lowest & 1,245 & 246 & 197.6 & 1.00 (reference) & \multirow{3}{*}{$<0.001$} & 1,699 & 434 & 255.4 & 1.00 (reference) & \multirow{4}{*}{$<0.001$} \\
\hline & Middle & 1,243 & 175 & 140.8 & $0.67(0.54-0.82)$ & & 1,700 & 319 & 187.6 & $0.67(0.57-0.79)$ & \\
\hline & Highest & 1,243 & 121 & 97.3 & $0.44(0.35-0.55)$ & & 1,699 & 225 & 132.4 & $0.45(0.37-0.53)$ & \\
\hline & Lowest & 1,243 & 222 & 178.6 & 1.00 (reference) & \multirow{3}{*}{$<0.001$} & 1,699 & 375 & 220.7 & 1.00 (reference) & \\
\hline \multirow[t]{2}{*}{ Cardiorespiratory fitness } & Middle & 1,245 & 172 & 138.2 & $0.78(0.60-1.00)$ & & 1,698 & 326 & 192.0 & $0.77(0.63-0.95)$ & \multirow[t]{2}{*}{$<0.001$} \\
\hline & Highest & 1,243 & 148 & 119.1 & $0.58(0.45-0.78)$ & & 1,701 & 277 & 162.8 & $0.68(0.55-0.84)$ & \\
\hline
\end{tabular}

CI, confidence interval.

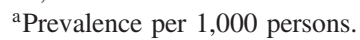


Ohta $\mathrm{T}$, et al.

Table 3. Associations of knee extensor muscle strength or cardiorespiratory fitness with low bone stiffness tendency

\begin{tabular}{|c|c|c|c|c|}
\hline & \multicolumn{3}{|c|}{ Knee extensor muscle strength } & \multirow{2}{*}{$P$ for trend } \\
\hline & Lowest & Middle & Highest & \\
\hline \multicolumn{5}{|l|}{ Men } \\
\hline Age adjusted & 1.00 (reference) & $0.69(0.55-0.84)$ & $0.45(0.36-0.57)$ & $<0.001$ \\
\hline Multivariable adjusted $^{\mathrm{a}}$ & 1.00 (reference) & $0.65(0.54-0.79)$ & $0.41(0.36-0.46)$ & $<0.001$ \\
\hline Mutual adjusted $^{\mathrm{b}}$ & 1.00 (reference) & $0.65(0.52-0.81)$ & $0.42(0.32-0.53)$ & $<0.001$ \\
\hline \multicolumn{5}{|l|}{ Women } \\
\hline Age adjusted & 1.00 (reference) & $0.94(0.79-1.12)$ & $0.86(0.71-1.05)$ & 0.145 \\
\hline Multivariable adjusted $^{\mathrm{a}}$ & 1.00 (reference) & $0.79(0.66-0.95)$ & $0.62(0.50-0.76)$ & $<0.001$ \\
\hline Mutual adjusted $^{\mathrm{b}}$ & 1.00 (reference) & $0.79(0.66-0.95)$ & $0.62(0.50-0.77)$ & $<0.001$ \\
\hline \multicolumn{5}{|l|}{ Pre-menopause } \\
\hline Age adjusted & 1.00 (reference) & $0.35(0.09-1.35)$ & $0.49(0.15-1.55)$ & 0.343 \\
\hline Multivariable adjusted $^{\mathrm{a}}$ & 1.00 (reference) & $0.31(0.08-1.20)$ & $0.30(0.09-1.02)$ & 0.090 \\
\hline Mutual adjusted $^{\mathrm{b}}$ & 1.00 (reference) & $0.30(0.08-1.21)$ & $0.31(0.09-1.05)$ & 0.100 \\
\hline \multicolumn{5}{|l|}{ Post-menopause } \\
\hline Age adjusted & 1.00 (reference) & $0.95(0.80-1.13)$ & $0.89(0.73-1.09)$ & 0.258 \\
\hline Multivariable adjusted $^{\mathrm{a}}$ & 1.00 (reference) & $0.81(0.67-0.97)$ & $0.63(0.51-0.78)$ & $<0.001$ \\
\hline \multirow[t]{3}{*}{ Mutual adjusted ${ }^{\mathrm{b}}$} & 1.00 (reference) & $0.80(0.67-0.97)$ & $0.63(0.51-0.78)$ & $<0.001$ \\
\hline & \multicolumn{3}{|c|}{ Cardiorespiratory fitness } & \multirow{2}{*}{$P$ for trend } \\
\hline & Lowest & Middle & Highest & \\
\hline \multicolumn{5}{|l|}{ Men } \\
\hline Age adjusted & 1.00 (reference) & $0.80(0.61-1.02)$ & $0.61(0.46-0.81)$ & $<0.001$ \\
\hline Multivariable adjusted $^{\mathrm{a}}$ & 1.00 (reference) & $0.74(0.55-0.97)$ & $0.45(0.33-0.62)$ & $<0.001$ \\
\hline Mutual adjusted ${ }^{\mathrm{b}}$ & 1.00 (reference) & $0.75(0.63-0.88)$ & $0.47(0.36-0.62)$ & $<0.001$ \\
\hline \multicolumn{5}{|l|}{ Women } \\
\hline Age adjusted & 1.00 (reference) & $1.02(0.81-1.28)$ & $1.25(0.98-1.61)$ & 0.085 \\
\hline Multivariable adjusted $^{\mathrm{a}}$ & 1.00 (reference) & $0.89(0.70-1.12)$ & $0.99(0.77-1.23)$ & 0.899 \\
\hline Mutual adjusted $^{\mathrm{b}}$ & 1.00 (reference) & $0.91(0.72-1.15)$ & $1.02(0.79-1.31)$ & 0.924 \\
\hline \multicolumn{5}{|l|}{ Pre-menopause } \\
\hline Age adjusted & 1.00 (reference) & $1.60(0.33-7.69)$ & $0.94(0.16-5.74)$ & 0.875 \\
\hline Multivariable adjusted $^{\mathrm{a}}$ & 1.00 (reference) & $1.50(0.29-8.16)$ & $0.65(0.10-4.28)$ & 0.529 \\
\hline Mutual adjusted $^{\mathrm{b}}$ & 1.00 (reference) & $1.69(0.31-9.26)$ & $0.74(0.11-5.02)$ & 0.632 \\
\hline \multicolumn{5}{|l|}{ Post-menopause } \\
\hline Age adjusted & 1.00 (reference) & $0.96(0.77-1.21)$ & $1.23(0.97-0.57)$ & 0.111 \\
\hline Multivariable adjusted $^{\mathrm{a}}$ & 1.00 (reference) & $0.93(0.74-1.18)$ & $1.03(0.81-1.32)$ & 0.851 \\
\hline Mutual adjusted $^{\mathrm{b}}$ & 1.00 (reference) & $0.96(0.76-1.21)$ & $1.05(0.82-1.35)$ & 0.704 \\
\hline
\end{tabular}

Values are expressed as odds ratio (95\% confidence interval).

aAdditionally adjusted smoking status, drinking habits, body mass index, systolic blood pressure, and menopause (for women only).

${ }^{\mathrm{b}}$ Mutually adjusted cardiorespiratory fitness or knee extensor muscle strength plus variables in multivariable adjustment ${ }^{\mathrm{a}}$.

An inverse association between knee extensor muscle strength and low bone stiffness tendency was observed after adjusting for potential confounders, such as age, smoking status, drinking habits, BMI, systolic blood pressure, menopause (for women only), and CRF in both sexes ( $P$ for linear trend $<0.001$ for both). Meanwhile, CRF and low bone stiffness tendency showed an inverse association in men $(P$ for linear trend $<0.001)$, but not in pre-menopausal, post-menopausal, and all women $(P$ for linear trend $=0.634,0.841$, and 0.924 , respectively), as shown in Table 3. Both knee extensor muscle strength and CRF had no interactive association with low bone stiffness tendency in both sexes (men; $P=0.836$, all women; $P=0.700$, post-menopausal women; $P=0.615)$, as shown in Table 4 .

\section{DISCUSSION}

Our primary findings were as follows. First, no interactive association between knee extensor muscle strength and CRF was found for low bone stiffness tendency in both sexes. Second, higher knee extensor muscle strength was associated with a lower prevalence of low bone stiffness tendency in both sexes, independent of CRF. Third, higher CRF was associated with a lower prevalence of low bone stiffness tendency in men but not in women, independent of knee extensor muscle strength. These findings suggest that a combination of muscle strength and CRF may carry an additive benefit, not synergistic, for bone health. However, the association between CRF and bone health may not be evident in adult Japanese women.

Contrary to our expectation, the knee extensor muscle strength and $\mathrm{CRF}$ had no interactive association with bone stiffness. Hence, each of the two physical fitness elements is independently or additively, not synergistically, associated with bone health. As described in the subsequent paragraphs, the possible mechanisms of muscle strength or CRF to bone strength may be different. However, it is unknown whether the two different pathways interfere with or work synergistically with each other, due to lack of findings from basic mechanistic studies. A previous randomized controlled trial has revealed that resistance exercise and a combination of resistance and aerobic exercise could prevent bone loss compared to aerobic exercise alone during a weight loss program. ${ }^{27}$ This finding supports the notion that a combined aerobic and resistance exercise may be recommended 
Table 4. Interacting association of knee extensor muscle strength and cardiorespiratory fitness with low bone stiffness tendency

\begin{tabular}{|c|c|c|c|c|c|c|}
\hline & Participant & Frequency & Prevalence $^{\mathrm{a}}$ & Odds ratio $(95 \% \mathrm{CI})^{\mathrm{b}}$ & Combined odds ratio $(95 \% \mathrm{CI})^{\mathrm{c}}$ & $\begin{array}{l}P \text { for } \\
\text { interaction }\end{array}$ \\
\hline Men & & & & & & 0.836 \\
\hline \multicolumn{7}{|l|}{ Lowest cardiorespiratory fitness } \\
\hline Lowest knee extensor muscle strength & 703 & 148 & 210.5 & 1.00 (reference) & 1.00 (reference) & \\
\hline Middle knee extensor muscle strength & 680 & 112 & 164.7 & $0.76(0.51-0.99)$ & $0.71(0.51-0.99)$ & \\
\hline Highest knee extensor muscle strength & 481 & 55 & 114.3 & $0.39(0.26-0.58)$ & $0.39(0.27-0.58)$ & \\
\hline \multicolumn{7}{|l|}{ Highest cardiorespiratory fitness } \\
\hline Lowest knee extensor muscle strength & 542 & 98 & 180.8 & 1.00 (reference) & $0.70(0.48-1.02)$ & \\
\hline Middle knee extensor muscle strength & 563 & 63 & 111.9 & $0.56(0.54-0.87)$ & $0.39(0.26-0.58)$ & \\
\hline Highest knee extensor muscle strength & 762 & 66 & 86.6 & $0.44(0.30-0.65)$ & $0.31(0.22-0.44)$ & \\
\hline Women & & & & & & 0.700 \\
\hline \multicolumn{7}{|l|}{ Lowest cardiorespiratory fitness } \\
\hline Lowest knee extensor muscle strength & 1,037 & 267 & 257.5 & 1.00 (reference) & 1.00 (reference) & \\
\hline Middle knee extensor muscle strength & 902 & 177 & 196.2 & $0.75(0.58-0.96)$ & $0.77(0.60-1.00)$ & \\
\hline Highest knee extensor muscle strength & 612 & 96 & 156.9 & $0.61(0.43-0.86)$ & $0.66(0.47-0.92)$ & \\
\hline \multicolumn{7}{|l|}{ Highest cardiorespiratory fitness } \\
\hline Lowest knee extensor muscle strength & 663 & 161 & 242.8 & 1.00 (reference) & $1.05(0.76-1.46)$ & \\
\hline Middle knee extensor muscle strength & 798 & 145 & 181.7 & $0.86(0.62-1.18)$ & $0.85(0.62-1.16)$ & \\
\hline Highest knee extensor muscle strength & 1,088 & 132 & 121.3 & $0.64(0.45-0.91)$ & $0.62(0.46-0.83)$ & \\
\hline Pre-menopause & & & & & & 0.373 \\
\hline \multicolumn{7}{|l|}{ Lowest cardiorespiratory fitness } \\
\hline Lowest knee extensor muscle strength & 79 & 4 & 50.6 & 1.00 (reference) & 1.00 (reference) & \\
\hline Middle knee extensor muscle strength & 137 & 2 & 14.6 & $0.28(0.01-7.37)$ & $0.44(0.03-7.16)$ & \\
\hline Highest knee extensor muscle strength & 170 & 1 & 5.9 & $0.05(0.00-1.41)$ & $0.12(0.01-2.45)$ & \\
\hline \multicolumn{7}{|l|}{ Highest cardiorespiratory fitness } \\
\hline Lowest knee extensor muscle strength & 70 & 3 & 42.9 & 1.00 (reference) & $0.87(0.04-20.44)$ & \\
\hline Middle knee extensor muscle strength & 195 & 2 & 10.3 & $0.05(0.00-2.06)$ & $0.04(0.00-2.38)$ & \\
\hline Highest knee extensor muscle strength & 388 & 7 & 18.0 & $0.44(0.06-3.12)$ & $0.39(0.03-4.48)$ & \\
\hline Post-menopause & & & & & & 0.615 \\
\hline \multicolumn{7}{|l|}{ Lowest cardiorespiratory fitness } \\
\hline Lowest knee extensor muscle strength & 958 & 265 & 276.6 & 1.00 (reference) & 1.00 (reference) & \\
\hline Middle knee extensor muscle strength & 765 & 180 & 235.3 & $0.76(0.59-0.98)$ & $0.78(0.61-1.01)$ & \\
\hline Highest knee extensor muscle strength & 441 & 94 & 213.2 & $0.64(0.45-0.90)$ & $0.68(0.49-0.96)$ & \\
\hline \multicolumn{7}{|l|}{ Highest cardiorespiratory fitness } \\
\hline Lowest knee extensor muscle strength & 592 & 162 & 273.6 & 1.00 (reference) & $1.05(0.76-1.44)$ & \\
\hline Middle knee extensor muscle strength & 603 & 135 & 223.9 & $0.88(0.64-1.22)$ & $0.88(0.65-1.19)$ & \\
\hline Highest knee extensor muscle strength & 701 & 123 & 175.5 & $0.64(0.45-0.91)$ & $0.62(0.46-0.84)$ & \\
\hline
\end{tabular}

CI, confidence interval.

${ }^{\text {aP }}$ Prevalence per 1,000 persons.

bAdjusted for age, smoking status, drinking habits, body mass index, systolic blood pressure, and menopause (for women only).

${ }^{\mathrm{c}}$ Using the lowest cardiorespiratory fitness and the lowest knee extensor muscle strength as reference.

to prevent a loss of musculoskeletal function. ${ }^{28}$ This may be consistent with our results.

Knee extensor muscle strength was positively associated with bone stiffness after adjusting for CRF in both sexes. According to Wolff's law, theories have indicated the potential of the bone to adapt its remodeling response to external stressors. ${ }^{29}$ It is assumed that knee extensor muscle strength may play the role of an external stressor to improve bone stiffness. Meanwhile, another possible mechanism of association between muscle strength and bone stiffness is related to muscle functioning as an endocrine organ that secretes myokines. Previous physiological studies have reported that muscles can play a role in secreting myokines that activate bone metabolism, such as myostatin, insulin-like growth factor (IGF)-1, and IGF-2. ${ }^{30}$ A previous observational study has reported an association between muscle strength and nonadjacent bones. ${ }^{31}$ However, the details of this association remain unclear, so further investigations into the mechanisms are warranted. Because bone mineral density has been highly correlated with bone stiffness, ${ }^{13,14}$ these are reflected in our study's results, as knee extensor muscle strength was associated with bone stiffness after adjusting for confounders.

CRF was also deemed beneficial for bone health in men. A previous large-scale observational study has showed similar trends as this study. ${ }^{32}$ CRF partially reflects one's physical activity level and has been utilized as an index of health outcome. $^{33}$ Because physical activity produces mechanical loading to the musculoskeletal system, which enhances osteocyte activation and bone resorption and formation, the American College of Sport Medicine currently recommends weightbearing physical activities. ${ }^{34}$ This study identified a sex difference in the association between CRF and bone stiffness (ie, no association in women). From a biological viewpoint, one possible mechanism for bone loss could be greater age-dependent loss of estrogen in women, which reduces estrogen receptor and osteogenic response. ${ }^{35}$ Accordingly, no association between CRF and bone health was observed in women, as in a previous study. ${ }^{36}$

This study has several noteworthy strengths. First, we analyzed a large-scale population of participants across a wide age range 
( $\geq 45$ years old). A difference in the age of onset between men and women of osteoporosis caused by lowering bone strength was observed. ${ }^{19}$ The number of patients with osteoporosis drastically increases after menopause for women and after the age of 60 for men. ${ }^{19}$ Second, we reduced potential biases by using multiple imputation methods. ${ }^{21}$ Third, this study expanded the body of knowledge in this area by examining the joint associations between knee extensor muscle strength and CRF with bone stiffness. Fourth, we used an isokinetic knee extensor muscle strength dynamometer, which enabled precise measurements. Although it requires expert skills, isokinetic muscle strength that reflects active physical activity could be assessed with accuracy. ${ }^{37}$

Meanwhile, there are also several limitations. First, causal associations among knee extensor muscle strength, CRF, and bone stiffness could not be assessed owing to the cross-sectional nature of the study. Second, there was no information on habitual dietary intake, such as protein, which could positively contribute to bone mineral density. ${ }^{38}$ Thus, the risk of residual confounding by dietary habits may exist in the results. Third, there was a lack of information on the use of osteoporosis medication and the history of surgery. Hence, bone stiffness could be underestimated. Finally, there was a lack of information on habitual physical activity. As half of the variances in CRF are accounted for by one's genotype, CRF is not a direct marker of physical activity, although it may reflect an aspect of physical activity. ${ }^{39}$ Therefore, precise assessments of physical activity are preferable.

\section{Conclusion}

We found an additive association between knee extensor muscle strength and CRF for bone health. Moreover, an inverse association existed between knee extensor muscle strength and low bone stiffness tendency in both sexes. Accordingly, maintaining not only higher knee extensor muscle strength, but also higher CRF may be important to prevent or delay the onset of osteoporosis. From a practical viewpoint, these results suggest that middle-aged or older women and men should be encouraged to participate in high-intensity physical activities with mechanical loading, such as running, jumping rope, and resistance exercise. Further longitudinal studies are needed to investigate the relationship between physical fitness and bone status.

\section{ACKNOWLEDGMENTS}

We thank the SPS participants and staff members at the Yokohama Sports Medical Center. We are also deeply grateful to Prof Susumu Sawada (Waseda University) for providing helpful comments. We would like to thank Editage (www. editage.com) for English language editing.

Author contributions: T.O. and H.S. designed the study; T.O., W.F. and J.N. collected the data; T.O. and W.F. analyzed the data; T.O. and H.S. wrote the first draft; J.N. and N.I. oversaw the entire project. All authors reviewed and contributed critical revisions of the manuscript and approved the final version of the manuscript.

Funding: This research was financially supported in part by an internal operational grant from the Tokyo Metropolitan Institute of Gerontology.

Conflicts of interest: As described above, the cost of English language editing is covered by an internal operational grant of TMIG, where H.S. works as a senior investigator. The other authors declare no conflict of interest.

\section{APPENDIX A. SUPPLEMENTARY DATA}

Supplementary data related to this article can be found at https:// doi.org/10.2188/jea.JE20200581.

\section{REFERENCES}

1. Tsuda T. Epidemiology of fragility fractures and fall prevention in the elderly. Curr Orthop Pract. 2017;28(6):580-585.

2. Qu X, Huang X, Jin F, et al. Bone mineral density and all-cause, cardiovascular and stroke mortality: a meta-analysis of prospective cohort studies. Int J Cardiol. 2013;166(2):385-393.

3. NIH Consensus Development Panel on Osteoporosis Prevention, Diagnosis, and Therapy. Osteoporosis Prevention, Diagnosis, and Therapy. JAMA. 2001;285(6):785-795.

4. Mohd-Tahir NA, Li SC. Economic burden of osteoporosis-related hip fracture in Asia: a systematic review. Osteoporos Int. 2017; 28(7):2035-2044.

5. Glüer CC. Quantitative ultrasound techniques for the assessment of osteoporosis: expert agreement on current status. The International Quantitative Ultrasound Consensus Group. J Bone Miner Res. 1997; 12(8):1280-1288.

6. Orimo H, Nakamura T, Hosoi T, et al. Japanese 2011 guidelines for prevention and treatment of osteoporosis-executive summary. Arch Osteoporos. 2012;7(1-2):3-20.

7. DeFina LF, Leonard D, Willis BL, et al. High cardiorespiratory fitness is associated with reduced risk of low bone density in postmenopausal women. J Womens Heal. 2016;25(10):1073-1080.

8. Kamiya K, Kajita E, Tachiki T, et al. Association between hand-grip strength and site-specific risks of major osteoporotic fracture: results from the Japanese Population-based Osteoporosis Cohort Study. Maturitas. 2019;130(September):13-20.

9. Bohannon RW, Magasi SR, Bubela DJ, Wang YC, Gershon RC Grip and knee extension muscle strength reflect a common construct among adults. Muscle Nerve. 2012;46(4):555-558.

10. Menant JC, Weber F, Lo J, et al. Strength measures are better than muscle mass measures in predicting health-related outcomes in older people: time to abandon the term sarcopenia? Osteoporos Int. 2017;28(1):59-70.

11. Manini TM, Visser M, Won-Park S, et al. Knee extension strength cutpoints for maintaining mobility. J Am Geriatr Soc. 2007;55(3): 451-457.

12. Suzuki T, Kim H, Yoshida H, Ishizaki T. Randomized controlled trial of exercise intervention for the prevention of falls in community-dwelling elderly Japanese women. J Bone Miner Metab. 2004;22(6):602-611.

13. Tsuda-Futami E, Hans D, Njeh CF, et al. An evaluation of a new gel-coupled ultrasound device for the quantitative assessment of bone. Br J Radiol. 1999;72:691-700.

14. Greenspan SL, Bouxsein ML, Melton ME, et al. Precision and discriminatory ability of calcaneal bone assessment technologies. J Bone Miner Res. 1997;12(8):1303-1313.

15. Morita R, Yamamoto I, Yuu I, et al. Quantitative ultrasound for the assessment of bone status. Osteoporos Int. 1997;7(Suppl 3):S128S134.

16. Japan Medical Journal. Osteoporosis Prevention Mannual by the Health and Medical Service Law for the Aged/Elderly (in Japanese); 2000.

17. Ohta T, Nagashima J, Sasai H, Ishii N. Relationship between cardiorespiratory fitness, body mass index, and incidence of dyslipidemia among Japanese women: a cohort study. Int J Environ Res Public Health. 2019;16:4647.

18. Miyashita M, Mutoh Y, Yoshioka N, Sadamoto T. PWC75\% HRmax: a measure of aerobic work Capacity. Sports Med. 1985; 2(3):159-164

19. Vilayphiou N, Boutroy S, Sornay-Rendu E, Van Rietbergen B, Chapurlat R. Age-related changes in bone strength from HR-pQCT derived microarchitectural parameters with an emphasis on the role of cortical porosity. Bone. 2016;83:233-240. 
20. Sterne JAC, White IR, Carlin JB, et al. Multiple imputation for missing data in epidemiological and clinical research: potential and pitfalls. BMJ. 2009;338:b2393.

21. White IR, Royston P, Wood AM. Multiple imputation using chained equations: issues and guidance for practice. Stat Med. 2011;30(4): 377-399.

22. Lane NE. Epidemiology, etiology, and diagnosis of osteoporosis. Am J Obstet Gynecol. 2006;194(2 SUPPL.):S3-S11.

23. Bijelic R, Milicevic S, Balaban J. Risk factors for osteoporosis in postmenopausal women. Med Arch. 2017;71(1):25-28.

24. Gaddini GW, Turner RT, Grant KA, Iwaniec UT. Alcohol: a simple nutrient with complex actions on bone in the adult skeleton. Alcohol Clin Exp Res. 2016;40(4):657-671.

25. Kalem MN, Kalem Z, Akgun N, Bakırarar B. The relationship between postmenopausal women's sclerostin levels and their bone density, age, body mass index, hormonal status, and smoking and consumption of coffee and dairy products. Arch Gynecol Obstet. 2017;295(3):785-793

26. Ye Z, Lu H, Liu P. Association between essential hypertension and bone mineral density: a systematic review and meta-analysis. Oncotarget. 2017;8(40):68916-68927.

27. Armamento-Villareal R, Aguirre L, Waters DL, Napoli N, Qualls C, Villareal DT. Effect of aerobic or resistance exercise, or both, on bone mineral density and bone metabolism in obese older adults while dieting: a randomized controlled trial. J Bone Miner Res. 2020; 35(3):430-439.

28. Villareal DT, Aguirre L, Gurney AB, et al. Aerobic or resistance exercise, or both, in dieting obese older adults. $N$ Engl J Med. 2017; 376(20):1943-1955

29. Brand RA. Biographical sketch: julius wolff, 1836-1902. Clin Orthop Relat Res. 2010;468(4):1047-1049.

30. Kaji H. Effects of myokines on bone. Bonekey Rep. 2016;5:826.
31. Luo Y, Jiang K, He M. Association between grip strength and bone mineral density in general US population of NHANES 2013-2014. Arch Osteoporos. 2020;15(1):47.

32. Wainstein HM, Feldman M, Shen CL, et al. The relationship between cardiorespiratory fitness and bone mineral density in men: a cross-sectional study. Mayo Clin Proc. 2016;91(6):726-734.

33. Haskell WL, Lee IM, Pate RR, et al. Physical activity and public health: updated recommendation for adults from the American College of Sports Medicine and the American Heart Association. Med Sci Sports Exerc. 2007;39(8):1423-1434.

34. Kohrt WM, Bloomfield SA, Little KD, Nelson ME, Yingling VR; American College of Sports Medicine. American College of Sports Medicine Position Stand: Physical activity and bone health. Med Sci Sports Exerc. 2004;36(11):1985-1996.

35. Lang TF. The bone-muscle relationship in men and women. J Osteoporos. 2011;2011:702735.

36. Kemmler W, Weineck J, Kalender WA, Engelke K. The effect of habitual physical activity, non-athletic exercise, muscle strength, and VO2max on bone mineral density is rather low in early postmenopausal osteopenic women. J Musculoskelet Neuronal Interact. 2004;4(3):325-334.

37. Sööt T, Jürimäe T, Jürimäe J, Gapeyeva H, Pääsuke M. Relationship between leg bone mineral values and muscle strength in women with different physical activity. J Bone Miner Metab. 2005;23(5):401406.

38. Darling AL, Millward DJ, Torgerson DJ, Hewitt CE, Lanham-New SA. Dietary protein and bone health: a systematic review and metaanalysis. Am J Clin Nutr. 2009;90(6):1674-1692.

39. Bouchard C, An P, Rice T, et al. Familial aggregation of VO(2max) response to exercise training: results from the HERITAGE family study. J Appl Physiol (1985). 1999;87(3):1003-1008. 